\title{
Detection of Low Energy X-rays with High Efficiency and Spectral Resolution
}

\author{
Lothar Strüder ${ }^{1,3 *}$, Heike Soltau ${ }^{2}$, Adrian Niculae ${ }^{2}$, Stefan Aschauer ${ }^{1}$, Robert Hartmann ${ }^{1}$ and Jeff Davis ${ }^{2}$ \\ 1. PNSensor GmbH, Otto-Hahn-Ring 6, 81739 Munich, Germany. \\ 2. PNDetector GmbH, Otto-Hahn-Ring 6, 81739 Munich, Germany. \\ 3. University of Siegen, Walter-Flex-Str. 3, 57068 Siegen, Germany. \\ * Corresponding author: lothar.strueder@pnsensor.de
}

Spectroscopic and imaging information of low energy X-rays in the energy range of $50 \mathrm{eV}$ up to several $\mathrm{keV}$ is of great importance for material science and the analysis of biological samples. For example, in material science Li-ion battery research is in the focus of many studies or the analysis of diffraction patterns recorded at synchrotron or Free Electron Laser sources from atomic clusters or viruses. In many of those cases the detection of single X-rays in the above energy band is required as well as intensity distributions with high dynamic range of X-rays with known energy.

There are several ways of creating a spectroscopic image with very soft X-rays: (a) a high energy resolving area detector collects the $\mathrm{X}$-ray radiation stimulated by electrons scanning across the sample (SEM, TEM) or (b) a collimated beam of protons (PIXE) or (c) a weakly focused beam of X-rays excite the atoms in the sample leading to a fluorescence and/or a diffraction signal recorded by a 1D or 2D detection system [1]. Relevant specifications for the detectors are (1) high quantum efficiency, (2) very low read-out noise (3) high speed, high dynamic range and (4) long term stability and reproducibility.

The interaction of low energy X-rays with matter - in our case silicon - happens in a very thin layer of the radiation entrance window of the detector [2]. For X-rays of $150 \mathrm{eV}$ the absorption length in silicon is less than $50 \mathrm{~nm}$, at the Carbon edge it is $112 \mathrm{~nm}$ and it is still below $3 \mu \mathrm{m}$ at $1 \mathrm{keV}$. This fact confronts detector developers with a difficult task: The silicon volume where the incident radiation interacts with the silicon lattice should not affect the collection process of the few signal charges. At $277 \mathrm{eV}$, the $\mathrm{C} \mathrm{K \alpha}$ energy, only 75 electron-hole pairs are created and only 14 electron-hole pairs at the $\mathrm{Li} \mathrm{K}$ line at $53 \mathrm{eV}$. The typical total electronic noise figure of $2-3$ electrons (rms) still allows for a proper detection of the $\mathrm{Li} \mathrm{K}, \mathrm{Be} \mathrm{K}, \mathrm{B} \mathrm{K}$ and $\mathrm{C} \mathrm{K}$ lines as can be seen in Fig. 2. The statistical fluctuations during the ionization process, called Fano effect limit the best possible energy resolution of $\mathrm{C} \mathrm{K} \alpha$ to $24 \mathrm{eV}$. Adding an electronic noise of 2.5 electrons (rms) results in an increase of the best achievable energy resolution of $33 \mathrm{eV}$ (FWHM). The measured width of the $\mathrm{C} \mathrm{K} \alpha$ line is $42 \mathrm{eV}$ (FWHM). The increased line width is due to the entrance window effects as highlighted in Fig.1. A variety of interactions of the incident X-ray and the subsequently generated energetic electrons lead to no energy deposition or a partial energy deposition in the sensitive silicon volume. Trapping and recombination amplifies this effect. This is responsible for the flat shelf in the spectrum and the $\mathrm{Si}$ escape line. In addition, $\mathrm{Al}$ and Si fluorescence is seen in Fig.1. The quality of the entrance window can be described by the incomplete charge collection (ICC) as a function of the penetration depth of the radiation in the silicon. The ICC indicates how the quality of the radiation entrance window limits the performance of the low energy response. In high quality detectors the ICC is about $95 \%$ at the $\mathrm{Si}_{-} \mathrm{SiO}_{2}$ interface and goes up to $100 \%$ in $200 \mathrm{~nm}$ depth. The low energy spectra have been calculated by means of numerical device simulations and MonteCarlo simulations. The measured spectra are in excellent agreement with the simulations leading to a good understanding of the underlying physics [4]. This knowledge leads to a tailored implementation of the right radiation entrance window and enables the 
scientist to perform imaging and spectroscopic measurements at the low energy side of the X-ray spectrum.

\section{References:}

[1] J Davis et al., Microscopy and Microanalysis 22(S3), p. 102.

[2] R Hartmann et al., Nucl. Inst. and Meth. in Phys. Res., A 439(2), p. 216.

[3] I Ordavo et al., Nucl. Inst. and Meth. in Phys. Res., A 654(1), p. 250.

[4] L Strüder, Synchrotron light sources and FELs, Springer Reference Series (2016).
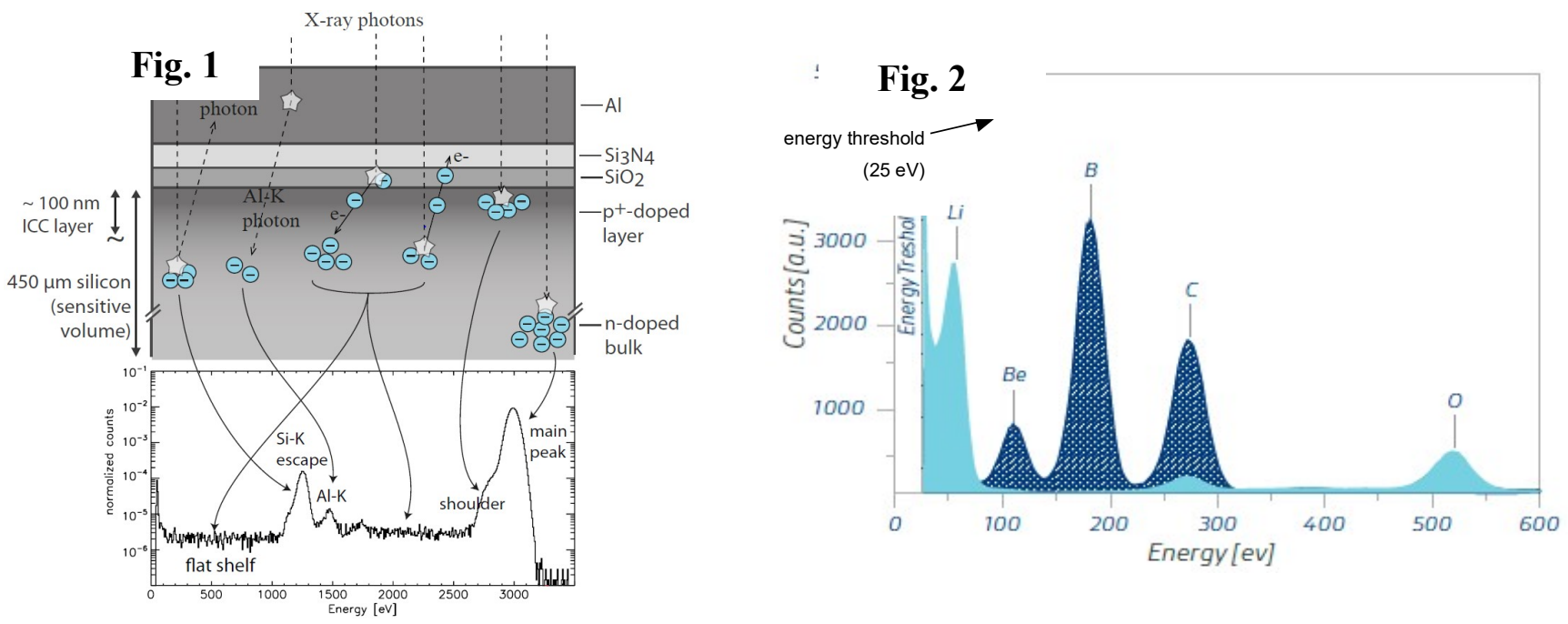

Figure 1. Model of a high-quality radiation entrance window. The X-rays have to pass through various layers of dielectrics and eventually Aluminum before they reach the sensitive volume in Silicon. Those processes lead to a complex response of the incident radiation, in this case $3 \mathrm{keV} \mathrm{X-rays} \mathrm{from} \mathrm{a} \mathrm{synchrotron.}$

Figure 2. Low energy X-ray spectrum recorded with an SDD from Li K up to O K. At C K the FWHM is $42 \mathrm{eV}$. Silicon drift detectors pnCCDs and the DePFET active pixel sensors are equipped with ultrathin radiation entrance windows designed for their respective use in their instruments. Not all the detector systems need a visible light blocking filter. It should be noted that the detectors used for this study (SDDs and pnCCDs) exhibit a Fano limited energy resolution for energies above $2 \mathrm{keV} \mathrm{[3].}$

Figure

3.

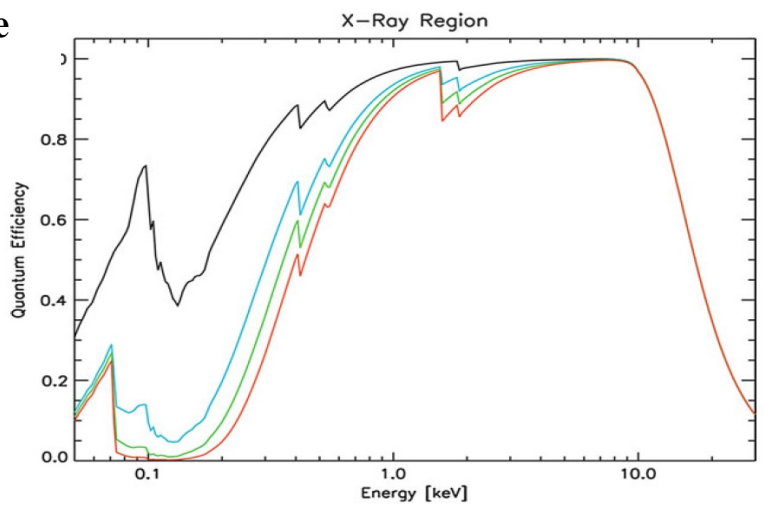

Figure 4.

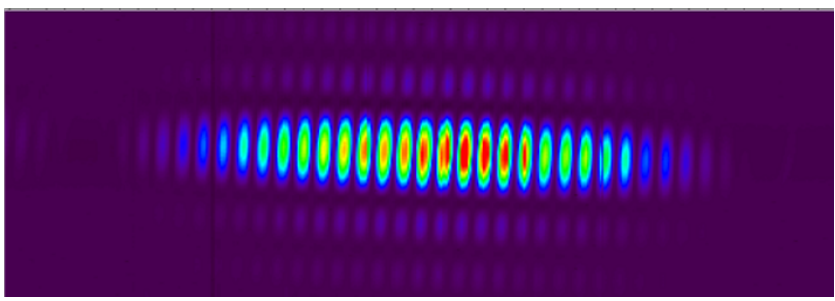

Figure 3. Quantum efficiency as a function of X-ray energy and different dielectrics and Al layers on the entrance window: black: $30 \mathrm{~nm}$ of $\mathrm{SiO}_{2}$ and $\mathrm{Si}_{3} \mathrm{~N}_{4}$, blue green and red, plus 50, 100 and $150 \mathrm{~nm}$ of $\mathrm{Al}$. Figure 4. Scattering pattern of a double slit irradiated with $90 \mathrm{eV}$ X-rays. The measured FWHM at $90 \mathrm{eV}$ was $38 \mathrm{eV}$. The quantum efficiency at $90 \mathrm{eV}$ was approximately $60 \%$. 